\title{
Long Time Efficacy and Safety of Microvascular Decom- pression Combined with Internal Neurolysis for Recurrent Trigeminal Neuralgia
}

\author{
Wenhao Zheng, ${ }^{1}$ Xiaoqiao Dong, ${ }^{2}$ Din Wang, ${ }^{2}$ Qiang $\mathrm{Hu}^{2}{ }^{2}$ Quan $\mathrm{Du}^{2}$ \\ The Fourth Clinical Medical College,' Zhejiang Chinese Medical University, Hangzhou, China \\ Department of Neurosurgery, The First People's Hospital of Hangzhou, Zhejiang University School of Medicine, Hangzhou, China
}

Objective : To explore the clinical efficacy and safety of microvascular decompression (MVD) combined with internal neurolysis (IN) in the treatment of recurrent trigeminal neuralgia (TN) after MVD.

Methods : Sixty-four patients with recurrent TN admitted to the hospital from January 2014 to December 2017 were divided into two groups according to the surgical method. Twenty-nine patients, admitted from January 2014 to December 2015, were treated with MVD alone, whereas 35 admitted from January 2016 to December 2017 were treated with MVD+IN. The postoperative efficacy, complications, and pain recurrence rate of the two groups were analyzed.

Results : The efficacy of the MVD+IN and MVD groups were $88.6 \%$ and $86.2 \%$, and the cure rates were $77.1 \%$ and $65.5 \%$ respectively. There was no statistically significant difference between the two groups $(p>0.05)$. The cure rate (83.3\%) of patients in the MVD+IN group, who were only found thickened arachnoid adhesions during the operation that could not be fully released, was significantly higher than that of the MVD group (30.0\%) $(p<0.05)$, while the efficacy $(91.7 \%$ vs. $70 \%)$ of the two groups was not statistically different $(p>0.05)$. For patients whose arachnoid adhesions were completely released, there had no significant difference $(p>0.05)$ in the efficacy ( $87 \%$ vs. $94.7 \%)$ and recurrence rate $(5.0 \%$ vs. $11.1 \%)$. The incidence of postoperative facial numbness $(88.6 \%)$ in the MVD+IN group was higher than that in the MVD group (10.3\%) $(p<0.01)$. The long-term incidence of facial numbness was not statistically significant ( $p>0.05)$. In the 18-36 months follow-up, the recurrence rate of patients in the MVD+IN group (9.7\%) and in the MVD group (16\%) were not statistically different $(p>0.05)$.

Conclusion : A retrospective comparison of patients with recurrent TN showed that both MVD and MVD combined with IN can effectively treat recurrent TN. Compared with MVD alone, MVD combined with IN can effectively improve the pain cure rate of patients with recurrent TN who have only severe arachnoid adhesions. The combination does not increase the incidence of longterm facial numbness and other complications.

Key Words : Trigeminal neuralgia · Microvascular decompression $\cdot$ Neurolysis $\cdot$ Arachnoid.

- Received : November 9, 2020 •Revised : December 15, 2020 •Accepted : March 26, 2021

- Address for reprints : Quan Du

Department of Neurosurgery, The First People's Hospital of Hangzhou, Zhejiang University School of Medicine, Huansha Road 261, Hangzhou, Zhejiang Province 310006, China

Tel : +86-13600511768, Fax : +86-56006972, E-mail : duquan76@163.com, ORCID : https://orcid.org/0000-0002-9871-0718

This is an Open Access article distributed under the terms of the Creative Commons Attribution Non-Commercial License (http://creativecommons.org/licenses/by-nc/4.0) which permits unrestricted non-commercial use, distribution, and reproduction in any medium, provided the original work is properly cited. 


\section{INTRODUCTION}

Trigeminal neuralgia (TN) is a pain syndrome characterized by repeated, paroxysmal, and transient severe pain in the trigeminal nerve distribution area. It is the most common clinical neurological disease. Microvascular decompression (MVD) is currently the best method for the treatment of TN and it has the longest duration of pain relief, with an efficacy of $>90 \%{ }^{11,12,18)}$. Clinically, some patients have recurrence of pain after MVD (time $>3$ months); they need medication that is sometimes ineffective. Literature suggests that MVD combined with internal neurolysis (IN) has a better effect on patients with recurrent TN after the first MVD surgery ${ }^{20)}$. In the current study, two surgical programs (MVD alone or combined with TN) were performed on patients with recurrent TN admitted to our hospital. The efficacy, complications, and recurrence of the two surgical programs were analyzed.

\section{MATERIALS AND METHODS}

The study was approved by the Institutional Review Board of Hangzhou First People's Hospital, China (IRB No. 2020240-01).

\section{Patient selection}

Sixty-four TN patients who had recurrent pain after MVD were admitted to the hospital from January 2014 to December 2017. The inclusion criteria are as follow : 1) patients with recurrent TN after MVD who do not respond to drugs or tolerate their side effects and do not accept any other invasive treatment such as sensory rhizotomy and Gamma knife radiosurgery; 2) based on the Burchiel classification of facial pain, patients with type $1 \mathrm{TN}$ characterized by episodic and idiopathic pain lasting several seconds, with pain-free intervals between attacks; 3 ) preoperative cranial magnetic resonance imaging to rule out intracranial space-occupying lesions or other secondary $\mathrm{TN}$; 4) those who have no serious heart, lung, liver, kidney, or other systemic diseases and can tolerate surgery; 5) patients, willing to accept MVD or MVD combined with IN, and to participate in clinical research follow-up; and 6) all patients involved in this study gave their informed consent. Institutional Review Board approval of our hospital was obtained for this study. The characteristics of patients with recurrent $\mathrm{TN}$ are outlined in Table 1.

\section{Surgical procedure}

Twenty-nine patients admitted from January 2014 to December 2015 were treated with MVD alone, whereas 35 admitted from January 2016 to December 2017 were treated with MVD+IN. The surgical procedure was performed as described : both groups of operations were performed by the same operation group. The patient takes the lateral position under general anesthesia, with the affected side facing upward,

Table 1. Clinical characteristics of the two groups of patients

\begin{tabular}{lccc}
\hline Factor & MVD+IN (n=35) & MVD (n=29) & 11/18 \\
\hline Male/femalue & $14 / 21$ & $61.62 \pm 1.68$ & 0.911 \\
Age (years) & $62.17 \pm 1.32$ & $19 / 10$ & 0.432 \\
Left/right & $22 / 13$ & $3(10.3)$ \\
Affected trigeminal division & & $6(20.7)$ \\
V1 & $4(11.4)$ & $4(13.8)$ \\
V2 & $7(20.0)$ & $6(20.7)$ \\
V3 & $5(14.3)$ & $7(24.1)$ \\
V1+V2 & $7(20.0)$ & $3(10.3)$ \\
V2+V3 & $9(25.7)$ & $31.21 \pm 1.63$ \\
V1+V2+V3 & $3(8.6)$ & \\
Pain relief time after the first MVD operation & $30.69 \pm 1.13$ & 0.730 \\
(months) & & \\
\hline
\end{tabular}

Values are presented as mean \pm standard deviation or number (\%). MVD : microvascular decompression, IN : internal neurolysis 
and the ipsilateral shoulder pulled downward to fully expose the operation area. Choose the original surgical incision, cut the scalp muscle layer by layer, and expose the bone window. Then remove the titanium plate, and expand the bone window if necessary. The upper edge extends to the transverse sinus whereas the lateral stretches to the edge of the sigmoid sinus. The dura mater is cut in a " $U$ " or " $V$ " shape to fully expose the area between the angle of the transverse sigmoid sinus and the main facial nerve. Slowly release the cerebrospinal fluid, fully cut the arachnoid membrane, fully expose the trigeminal nerve root under the microscope, and explore the responsible blood vessel at root entry zone (REZ). Cut off the Teflon cotton ball. Loosen the adhering arachnoid membrane, and re-insert a suitable size Teflon cotton ball between the responsible blood vessel and the trigeminal nerve root to ensure that the responsible blood vessel no longer oppresses or touches the trigeminal nerve root. If there is only severe arachnoid adhesion, release the arachnoid adhesion as much as possible, but excessive traction on peripheral nerves and blood vessels should be avoided, and then replace the previous Teflon felt. After the trigeminal nerve root and peripheral arachnoid are completely loosened, use a nerve comb knife to comb the trigeminal nerve root from REZ to petrous bone in 5-10 levels (Fig. 1). During the operation, the internal auditory canal, facial nerve, petrosal vein, and surrounding important structures must be protected. After the operation, the dura mater is sutured tightly, the bone flap is reset and fixed, and the muscle scalp is sutured layer by layer. Patients in the MVD group are not able to comb the trigeminal nerve root, and the rest of the steps are the same.

\section{Outcome measures and follow-up}

The surgical effect, postoperative complications, and recurrence rate were compared between the two groups. Reference was made to the Barrow Neurological Institute (BNI) pain intensity scoring criteria and the BNI facial numbness score (Table 2) to determine the postoperative effect ${ }^{15)}$. Outcome measures : cure, pain disappeared completely without medication (score 1); relief, occasional or some pain, which can be controlled without or with small doses of drugs (score 2 or 3); ineffective, no pain relief or pain cannot be adequately controlled with medication (score 4 or 5). Cure or relief is considered effective. Surgery complications include intracranial infection, facial numbness, tinnitus, hearing impairment, lower cranial nerve injury, cerebellar or brainstem hemorrhage, infarction, and death. Pain recurrence means that the pain reappears or is aggravated after relief (the increase of BNI pain intensity score more than one score), which occurs at least three months after surgery ${ }^{3)}$.

All patients in two groups were contacted by an independent observer who was not involved in patient care, through telephone. The follow-up ranged from 18 to 36 months.

\section{Statistical analysis}

Data analysis was performed using SPSS ver. 23.0 statistical software (IBM Corp, Armonk, NY, USA). The Kaplan-Meier survival curve was performed to determine the percentage of patients who were pain-free, off medications, after the operation. Univariate comparisons of continuous variables were compared using the Student t-test. The differences of proportion were compared by the chi-squared test. Those $p<0.05$ was considered statistically significant.
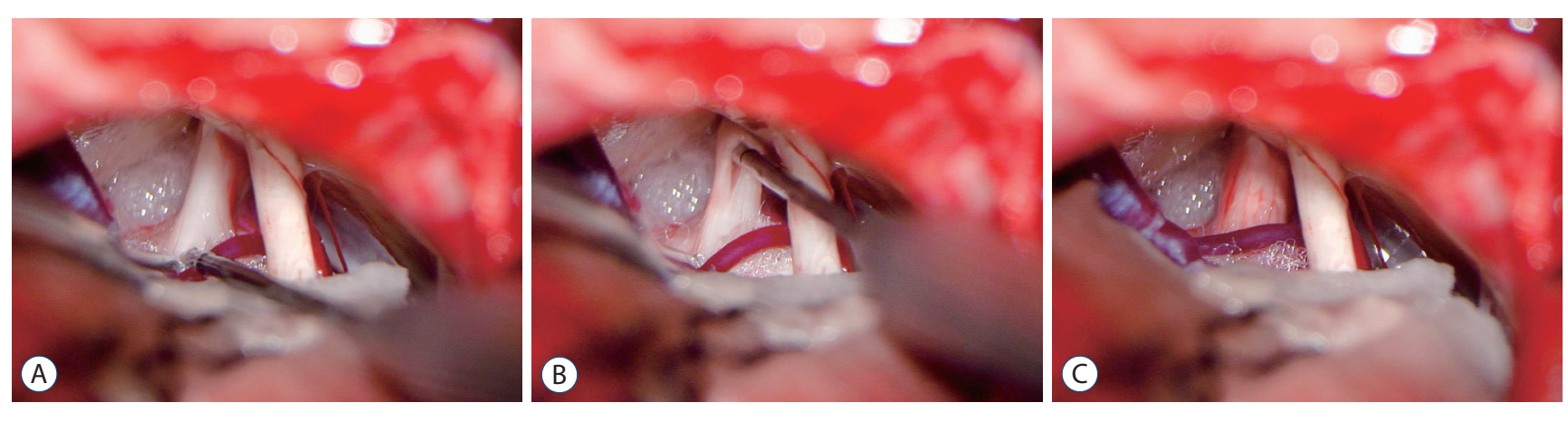

Fig. 1. Internal neurolysis during operation. A : Intraoperative view in patients with recurrent trigeminal neuralgia. B : The internal neurolysis procedure includes separating the trigeminal nerve longitudinally, using a nerve comb knife to comb the trigeminal nerve root from root entry zone to petrous bone in 5-10 levels. C: The final configuration of trigeminal nerve after internal neurolysis. 


\section{RESULTS}

\section{Demographics and clinical characteristics}

The average age of patients in the MVD+IN group was 62.17 years old, whereas the average age in the MVD group was 61.62. There were 14 males and 21 females in the MVD+IN group, and 11 males and 18 females in the MVD group. The average time of pain relief after the first MVD operation in the MVD+IN group was 30.69 months, whereas the average time in the MVD group was 31.21 months. The difference of average age, sex, and average time of pain relief after the first MVD between the two groups was not statistically significant $(p>0.05)$.

Table 2. Operative findings in MVD+IN group and MVD group

\begin{tabular}{lcc}
\hline & MVD+IN & MVD \\
\hline Dense arachnoid adhesions & $17(48.6)$ & $14(48.3)$ \\
Incomplete arachnoid release & $12(70.6)$ & $10(71.4)$ \\
Teflon felt displacement & $9(25.7)$ & $8(27.6)$ \\
New responsible vascular compression & $8(22.9)$ & $7(24.1)$ \\
Teflon granuloma & $1(2.8)$ & $0(0.0)$ \\
\hline
\end{tabular}

Values are presented as number (\%). MVD : microvascular decompression, IN : internal neurolysis

\section{Operative findings}

In the MVD+IN group, there were 17 cases with dense arachnoid adhesions, nine with the displacement of Teflon felt, eight with new responsible vascular compression, and one with Teflon granuloma formation. In the MVD group, there were 14 cases with dense arachnoid adhesions during the operation, eight with the displacement of Teflon felt, seven with new responsible vascular compression, and no Teflon granuloma formation. There were 12 cases (70.6\%) and 10 cases $(71.4 \%)$ of the arachnoid with thickened adhesions that could not be completely released in the MVD+IN and the MVD group respectively (Table 2). The difference was not statistically significant $(p>0.05)$.

\section{Postoperative complications}

In the MVD+IN group, 31 cases (65.7\%) had numbness on the side of the operation, 27 cases had numbness disappear or decrease significantly in 6 months after the operation, and four cases had no obvious remission. In the MVD group, three cases $(10.3 \%)$ had numbness on the side of the operation, and the numbness disappeared or decreased significantly in three cases after 6 months. The incidence of facial numbness in the MVD+IN group was significantly higher than that in the MVD group $(p<0.01)$. The incidence of facial numbness in the two groups was not statistically significant in the longterm ( $>6$ months) postoperative period $(p>0.05)$. Five cases

Table 3. Comparison of the postoperative complications for patients who underwent MVD+IN or MVD for recurrent trigeminal neuralgia

\begin{tabular}{lccc}
\hline Complication & MVD+IN & MVD & p-value \\
\hline Herpes simplex & $5(14.3)$ & $3(10.3)$ & 0.719 \\
Facial numbness & $31(88.6)$ & $3(10.3)$ & $26(89.7)$ \\
$\quad$ No facial numbness & $4(11.4)$ & $3(10.3)$ \\
Mild facial numbness, not bothersome & $28(80.0)$ & $0(0.0)$ \\
Facial numbness, somewhat bothersome & $3(8.6)$ & $0(0.0)$ \\
Facial numbness, very bothersome & $0(0.0)$ & $0(0.0)$ \\
Long-term facial numbness (>6 months) & $4(11.4)$ & $0(0.0)$ \\
Intracranial infection & $0(0.0)$ & $0(0.0)$ \\
Tinnitus & $0(0.0)$ & $0(0.0)$ \\
Hearing impairment & $0(0.0)$ & $0(0.0)$ \\
Lower cranial nerve injury & $0(0.0)$ & $0(0.0)$ \\
Cerebellar or brainstem hemorrhage & $0(0.0)$ & $0(0.0)$ \\
Death & $0(0.0)$ & 1.000 \\
\hline
\end{tabular}

Values are presented as number (\%). MVD : microvascular decompression, IN : internal neurolysis 
(14.3\%) in the MVD+IN group developed herpetic angular cheilitis after the operation, three cases (10.3\%) in the MVD group developed herpetic angular cheilitis, and all recovered within 7 to 14 days after the operation. There was no statistically significant difference in the incidence of angular cheilitis ( $p>0.05)$. There were no cases of intracranial infection, tinnitus, hearing impairment, lower cranial nerve injury, cerebellar or brainstem hemorrhage, and death in both groups (Table 3).

\section{Efficacy and follow-up}

In the MVD+IN group, 27 cases of facial pain disappeared, five cases were relieved, three cases were ineffective. The efficacy was $88.6 \%$, the cure rate was $77.1 \%$ and the inefficiency was $11.4 \%$. In the MVD group, 19 cases were pain-free, four were relieved and four were ineffective. The efficacy was $86.2 \%$, the cure rate was $65.5 \%$, and the inefficiency was $13.8 \%$. There was no significant difference in efficacy and cure rate between the two groups ( $p>0.05$ ) (Table 4).

Among the 12 patients in the MVD+IN group who only had dense arachnoid adhesions and could not fully release the arachnoid adhesions during the operation, 10 cases of facial episodic pain disappeared, one case was relieved, one case was ineffective, the efficacy was $91.7 \%$, the cure rate was $83.3 \%$, and the inefficiency was $8.3 \%$. Among the 10 patients in the MVD group, three cases of facial pain disappeared, four cases were relieved, three cases were ineffective, the efficacy was
$70.0 \%$, the cure rate was $30.0 \%$, and the inefficiency was $30.0 \%$ (Table 4). The cure rate of patients with MVD+IN unable to fully release arachnoid adhesions was higher than that of the MVD group $(p<0.05)$, while the difference in the effica$c y$ and recurrence rate between the two groups was not significant $(p>0.05)$. As for patients whose arachnoid adhesions were completely released, the efficacy ( $87 \%$ vs. $94.7 \%)$ and recurrence rate (5.0\% vs. $11.1 \%$ ) between two groups had no sig-

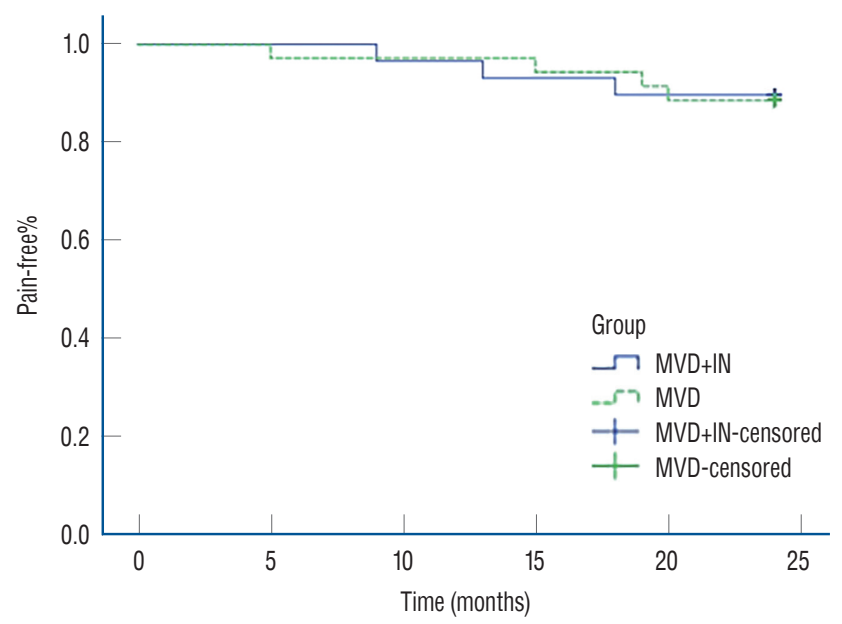

Fig. 2. Kaplan-Meier survival curves comparing the percentage of patients who were pain free off medications after MVD+IN (solid line) and MVD (dashed line). There were no significant differences between the two groups $(p>0.05)$. MVD : microvascular decompression, IN : internal neurolysis.

Table 4. Comparison of the clinical effect of two groups after operation

\begin{tabular}{|c|c|c|c|c|c|}
\hline & Cured & Improved & Effective & Ineffective & Recurrent \\
\hline \multicolumn{6}{|l|}{ All patients } \\
\hline MVD+IN & $27(77.1)$ & $4(11.4)$ & $31(88.6)$ & $4(11.4)$ & $3(9.7)$ \\
\hline MVD & $19(65.5)$ & $6(20.9)$ & $25(86.2)$ & $4(13.8)$ & $4(16.0)$ \\
\hline$p$-value & 0.303 & & 1.000 & & 0.761 \\
\hline \multicolumn{6}{|c|}{ Patients with ICRAA } \\
\hline MVD+IN & 10 (83.3) & $1(8.3)$ & $11(91.7)$ & $1(8.3)$ & $2(18.2)$ \\
\hline MVD & $3(30.0)$ & $4(40.0)$ & $7(70.0)$ & $3(30.0)$ & $2(28.6)$ \\
\hline$p$-value & 0.036 & & 1.000 & & 1.000 \\
\hline \multicolumn{6}{|c|}{ Patients with CRAA } \\
\hline MVD+IN & $17(74.0)$ & $3(13.0)$ & $20(87.0)$ & $3(13.0)$ & $1(5.0)$ \\
\hline MVD & $16(84.2)$ & $2(10.5)$ & $18(94.7)$ & $1(5.3)$ & $2(11.1)$ \\
\hline$p$-value & 0.655 & & 0.731 & & \\
\hline
\end{tabular}

Values are presented as number (\%). MVD : microvascular decompression, IN : internal neurolysis, ICRAA : the incomplete release of arachnoid adhesions, CRAA : the complete release of arachnoid adhesions 
nificant difference $(p>0.05)$ (Table 4).

The average follow-up duration was 27.2 months, ranging from 18 to 36 months. During the follow-up, three patients (9.7\%) in the MVD+IN group and four (16\%) in the MVD group recurred. There was no significant difference between the two groups $(p>0.05)$ (Table 4$)$. The Kaplan-Meier survival curve of the two groups of patients is shown in Fig. 2. The difference between the two groups was not statistically significant (log-rank test, $p>0.05$ ).

\section{DISCUSSION}

TN, also known as painful convulsions, was first reported by Dandy in $1929^{14)}$. It is most common in middle-aged and elderly female patients. The disease occurs frequently on the left side ${ }^{17)}$. Frequent and severe pain episodes often cause unbearable pain and seriously affect the quality of life of patients. The pathogenesis of TN is currently not clear, but most scholars believe that the trigeminal nerve REZ pulsates and is impacted by the dilated, elongated, tortuous, and hardened vertebral-basal artery vessels, causing changes in the demyelination of the trigeminal nerve. The most common responsible vessel is the superior cerebellar artery, while the petrosal vein is more common in venous compression ${ }^{9)}$.

Jannetta $^{7)}$ proposed MVD to treat TN based on the theory of vascular compression in 1967. MVD is currently the first choice for the treatment of TN, and has the advantages of good pain relief, low recurrence rate, and fewer side effects. However, $5-10 \%$ of TN patients experience pain again after receiving $\mathrm{MVD}^{1)}$. The reasons for the recurrence of $\mathrm{TN}$ are as follows : 1) dense arachnoid adhesions : in this study, $31 \mathrm{pa}-$ tients (48.4\%) who had recurrence after MVD surgery had thickened arachnoid membrane adhesions, without any responsible vascular compression at the trigeminal nerve root during the second surgical exploration. Feng et al. ${ }^{4)}$ reported that obvious arachnoid adhesions were seen in the first MVD placement of the Teflon felt and cerebellopontine angle (CPA) and even part of patients formed arachnoid cysts during the second surgical exploration ${ }^{22}$. The thickened arachnoid adhesions not only change the normal anatomical position of blood vessels and trigeminal nerve, but also cause compression and involvement on the trigeminal nerve, finally giving rise to the typical clinical manifestation of TN. 2) Teflon felt factors : in 17 patients (26.6\%), it was observed that the volume or position of the Teflon felt was inappropriate, falling off, and shifting during the exploration. The volume of Teflon was too small leading to insufficient decompression between the responsible vessels and nerve roots, the pulsating compression of blood vessels can still affect the trigeminal nerve through the Teflon, bringing about the pain recurrence; whereas too large can generate new oppression on the nerve roots. Improper position, shedding, and displacement of the Teflon felt causes the responsible vessel to recompress the trigeminal nerve root. 3) Responsible vascular compression : 15 patients (23.4\%) had a new responsible vascular compression on the REZ during the second surgical exploration. The new responsible blood vessels can be arteries, veins, or arteries combined with veins. It has been reported that patients with vein or arteriovenous combination as the responsible blood vessel are more likely to have pain recurrence after the first MVD. 4) Teflon felt granuloma formation : one patient (2.8\%) had the formation of Teflon felt granuloma the during operation. Inflammation occurs locally around the Teflon felt between nerves and vessels, brings about granuloma that adheres to nerves, blood vessels, or brainstem, and causes the trigeminal nerve root to be twisted and compressed ultimately. Gu and Zhao ${ }^{5)}$ reported that the incidence of Teflon granuloma after MVD was approximately 5\%. And 5) surgical factors : the surgical experience of the surgeon will affect the efficacy of the first MVD.

Carbamazepine is the initial choice for treatment for patients with recurrence of TN after the first $\mathrm{MVD}^{13)}$. Surgery is reconsidered only when the drug treatment is ineffective or patients cannot tolerate the side effects. Redo MVD is regarded as a good choice for patients with recurrent pain after the first $\mathrm{MVD}^{1,2,5)}$, and $67-83.3 \%$ of patients with pain recurrence can be cured for a long time. However, the curative effect of the redo MVD was lower than that of the first MVD ${ }^{6}$. There are two reasons as follows : 1) the adhesion between nerve fibers is severe due to long-term vascular compression and 2) the adhesion of the Teflon felt placed in the first MVD between trigeminal nerve and vessel cannot be completely removed. According to these reasons, IN may be the appropriate choice for recurrent TN. IN was reported by many scholars as the treatment for TN without vascular compression. The trigeminal nerve is combed from the REZ to the petrous bone in 5-10 levels of longitudinal nerves, which can separate the ad- 
hering trigeminal nerve fiber bundles, destroy the interconnection between trigeminal nerve fibers, and inhibit the abnormal nerve signal conduction ${ }^{10)}$. IN can also loosen dense arachnoid adhesions and Teflon felt attached to the surface of the trigeminal nerve, reducing the conduction of abnormal electrical signals. Zhang et al. ${ }^{20)}$ described 148 patients who underwent recurrent or persistent TN. The success rate of MVD plus IN at 1 year was $93.75 \%$, which was significantly higher than the success rate of simple MVD (78.95\%). In this study, the efficacy of the MVD+IN group was $88.6 \%$, and the cure rate was $77.1 \%$, which was not significantly different from that of the MVD group. However, the cure rate $(83.3 \%)$ of patients in the MVD+IN group who could not fully release the arachnoid adhesions during the operation was significantly higher than that of the MVD group (30.0\%). The results show that MVD combined with IN can improve the cure rate of patients with incomplete arachnoid adhesions. Therefore, MVD plus IN is performed in recurrent TN patients, whose thick arachnoid adhesions could not be fully released during the operation. However, if adhesions of arachnoid along the trigeminal nerve could be released thoroughly, simple MVD is sufficient for relieving the pain. The clinical outcomes of the two groups with their arachnoid adhesions completely released demonstrates that IN does not have to be performed in every case where the trigeminal nerve root and peripheral arachnoid are completely loosened.

The most common complication of MVD combined with IN is ipsilateral numbness ${ }^{10,19,20)}$. Most of the patients in this group $(88.6 \%)$ had different degrees of facial numbness on the surgical side after operation, which was much higher than that after MVD alone $(10.3 \%)(p<0.01)$. Facial numbness occurs mainly because the myelin sheath and some axons of nerve fibers are destroyed during the process of IN. Most of the axons can be retained as the "track" for remyelination and myelin can regenerate and restore nerves along the axons. This mild injury reversible action because of the self-repair of the nerve ${ }^{8,16,21)}$. Therefore, patients with numbness in the back of IN can be relieved to varying degrees after some time. Most patients can recover or relieve within 6 months post-surgery. Only a few patients still feel significant facial numbness, which may be related to the excessive combing of the trigeminal nerve. Herpes simplex is also one of the common postoperative complications, which is caused by herpes simplex virus infection. The virus can be latent in the sensory ganglion cells for a long time after it infects adults and can present clinically due to immunosuppression or other inducements. Most cases of herpes simplex virus infection are not serious and can heal spontaneously. The incidence of cerebellar injury and hemorrhage is generally $<1 \%$. To avoid cerebellar injury, the traction time and strength should be reduced, the intersection of the transverse and sigmoid sinuses exposed when opening the bone window, and the cerebrospinal fluid fully released before exploring CPA. The traction of the cerebellar hemisphere should be minimized during the operation, and if necessary, a dehydrating agent used to reduce intracranial pressure. The incidence of cerebrospinal fluid fistula is $3 \%$, mainly because the mastoid air chamber is opened during the operation without bone wax sealing or not tightly closed, and the dura mater and the incision are not tightly sutured. Strict and careful technique to close the incision and tightly sealing the open mastoid air chambers can reduce the incidences of cerebrospinal fluid fistulas after surgery.

\section{CONCLUSION}

A retrospective comparison of patients with recurrent $\mathrm{TN}$ showed that both MVD with IN and MVD can effectively treat recurrent TN. Compared with MVD alone, MVD combined with IN can effectively improve the pain cure rate of patients with recurrent $\mathrm{TN}$ who have only severe arachnoid adhesions. The combination does not increase the incidence of long-term facial numbness and other complications.

\section{Limitation}

There are several apparent limitations to this study. 1) The small sample size may influence the conclusions that can be drawn from statistical analysis. 2) The follow-up period of the two groups was short, and long-term complications and recurrence may be relatively underestimated. The possibility that the bias caused by the difference in the follow-up period between the two groups cannot be excluded.

\section{CONFLICTS OF INTEREST}

No potential conflict of interest relevant to this article was reported. 


\section{INFORMED CONSENT}

Informed consent was obtained from all individual participants included in this study.

\section{AUTHOR CONTRIBUTIONS}

\author{
Conceptualization : QD \\ Data curation : XD \\ Formal analysis : $\mathrm{QH}$ \\ Funding acquisition : DW \\ Methodology: WZ \\ Project administration : QD \\ Visualization : WZ \\ Writing - original draft : WZ \\ Writing - review \& editing : QD
}

\section{ORCID}

$\begin{array}{ll}\text { Wenhao Zheng } & \text { https://orcid.org/0000-0001-5590-6588 } \\ \text { Xiaoqiao Dong } & \text { https://orcid.org/0000-0003-2744-5459 } \\ \text { Din Wang } & \text { https://orcid.org/0000-0001-7552-2389 } \\ \text { Qiang Hu } & \text { https://orcid.org/0000-0002-6606-5347 } \\ \text { Quan Du } & \text { https://orcid.org/0000-0002-9871-0718 }\end{array}$

\section{References}

1. Bakker NA, Van Dijk JM, Immenga S, Wagemakers M, Metzemaekers JD : Repeat microvascular decompression for recurrent idiopathic trigeminal neuralgia. J Neurosurg 121 : 936-939, 2014

2. Cheng J, Meng J, Lei D, Hui X : Repeat microvascular decompression for patients with persistent or recurrent trigeminal neuralgia: prognostic factors and long-term outcomes. Medicine (Baltimore) 98 : e15167, 2019

3. Deng Z, Liu R, Liu Y, Wang Z, Yu Y, Zhang L : Factors that may affect delayed relief of trigeminal neuralgia after microneurosurgery and the long-term outcomes associated with delayed relief. J Pain Res 12 : 2817-2823, 2019

4. Feng BH, Wang XH, Li ST : Posterior fossa re-exploration for recurrent trigeminal neuralgia: operative findings and surgical techniques. J Craniofac Surg 29 : 1284-1286, 2018

5. Gu W, Zhao W : Microvascular decompression for recurrent trigeminal neuralgia. J Clin Neurosci 21 : 1549-1553, 2014
6. Hussain MA, Konteas A, Sunderland G, Franceschini P, Byrne P, OsmanFarah J, et al. : Re-exploration of microvascular decompression in recurrent trigeminal neuralgia and intraoperative management options. World Neurosurg 117 : e67-e74, 2018

7. Jannetta PJ : Arterial compression of the trigeminal nerve at the pons in patients with trigeminal neuralgia. J Neurosurg 26 Suppl : 159-162, 1967

8. Jie $H$, Xuanchen $Z$, Deheng $L$, Kun G, Fengyang $X$, Xiang $C$, et al. : The long-term outcome of nerve combing for trigeminal neuralgia. Acta Neurochir (Wien) 155 : 1703-1708; discussion 1707, 2013

9. Kakizawa Y, Seguchi T, Kodama K, Ogiwara T, Sasaki T, Goto T, et al. : Anatomical study of the trigeminal and facial cranial nerves with the aid of 3.0-tesla magnetic resonance imaging. J Neurosurg $108: 483-490$, 2008

10. Ko AL, Ozpinar A, Lee A, Raslan AM, McCartney S, Burchiel KJ : Longterm efficacy and safety of internal neurolysis for trigeminal neuralgia without neurovascular compression. J Neurosurg 122 : 1048-1057, 2015

11. Liao C, Zhang W, Yang M, Zhong W, Liu P, Li S : Microvascular decompression for trigeminal neuralgia: the role of mechanical allodynia. World Neurosurg $91:$ 468-472, 2016

12. Liu J, Wu G, Jiang Y, Li L, Wang D, Liu R : Relationship between arterial blood pressure during trigeminal nerve combing and surgical outcome in patients with trigeminal neuralgia. World Neurosurg 137 : e98-e105, 2020

13. Lu VM, Duvall JB, Phan K, Jonker BP : First treatment and retreatment of medically refractive trigeminal neuralgia by stereotactic radiosurgery versus microvascular decompression: a systematic review and metaanalysis. Br J Neurosurg 32 : 355-364, 2018

14. Patel SK, Liu JK : Overview and history of trigeminal neuralgia. Neurosurg Clin N Am 27 : 265-276, 2016

15. Rogers $C L$, Shetter $A G$, Fiedler JA, Smith $K A$, Han PP, Speiser BL : Gamma knife radiosurgery for trigeminal neuralgia: the initial experience of the Barrow Neurological Institute. Int J Radiat Oncol Biol Phys 47 : 1013-1019, 2000

16. Sabourin V, Mazza J, Garzon T, Head J, Ye D, Stefanelli A, et al. : Internal neurolysis with and without microvascular decompression for trigeminal neuralgia: case series. World Neurosurg 143 : e70-e77, 2020

17. Siqueira SR, Teixeira MJ, Siqueira JT : Clinical characteristics of patients with trigeminal neuralgia referred to neurosurgery. Eur J Dent 2009 : 207-212, 2009

18. Wu A, Doshi T, Hung A, Garzon-Muvdi T, Bender MT, Bettegowda C, et al. : Immediate and long-term outcomes of microvascular decompression for mixed trigeminal neuralgia. World Neurosurg 117 : e300-e307, 2018

19. Wu M, Jiang $X$, Niu C, Fu X: Outcome of internal neurolysis for trigeminal neuralgia without neurovascular compression and Its relationship with intraoperative trigeminocardiac reflex. Stereotact Funct Neurosurg 96 : 305-310, 2018

20. Zhang $X, X u L$, Zhao H, Tang YD, Zhu J, Yuan Y, et al. : Long-term efficacy of nerve combing for patients with trigeminal neuralgia and failed 
prior microvascular decompression. World Neurosurg 108 : 711-715, 2017

21. Zhao $H$, Zhang $X$, Tang $D$, Li $S$ : Nerve combing for trigeminal neuralgia without vascular compression. J Craniofac Surg 28 : e15-e16, 2017
22. Zhong J, Li ST, Zhu J, Guan HX, Zhou QM, Jiao W, et al. : A clinical analysis on microvascular decompression surgery in a series of 3000 cases.

Clin Neurol Neurosurg 114 : 846-851, 2012 